\title{
Gender is a risk factor in patients with gastroesophageal reflux disease
}

\author{
Hashem Fakhre Yaseri ${ }^{1 *}$ \\ Received: 07 Feb 2017 \\ Published: 8 Sep 2017
}

\begin{abstract}
Background: Prevalence of gastroesophageal reflux disease (GERD) has increased in the last decades, and it is now one of the most common chronic and recurrent diseases. The present study aimed at determining the frequency of gender (sex) and age in Iranian patients with GERD symptoms.

Methods: In this study, 803 patients aged 11 to 84 years, with erosive and nonerosive gastroesophageal reflux diseases, based on the questionnaire and esophagogastroduodenoscopy findings, participated. The female group was compared with the male group with respect to age, symptoms, esophageal injury, and hiatus hernia.

Results: Of the 803 participants, $60.5 \%(n=486)$ were female, and $69.2 \%(n=555)$ were younger than 50 years. Of those patients older than 50 years, $32.8 \%(\mathrm{n}=81)$ were female. Moreover, $31.0 \%(\mathrm{n}=249)$ of the patients had erosive esophagitis $(\mathrm{ERD})$, and $69.0 \%$ $(n=254)$ had normal esophageal mucosa (NERD). The female to male ratio was $1 / 1.06$ and 1.94/1 in ERD and NERD patients, respectively. Hiatal hernia was more prevalent in females than in males.

Conclusion: Nonerosive reflux disease, as a gastroesophageal reflux disease (GERD), was more common in females than in males. GERD became more prevalent with increase in age. Gender and hiatal hernias were 2 potential risk factors of GERD.
\end{abstract}

Keywords: Esophageal Disease, Gastroesophageal Reflux Disease, Esophageal Reflux, Heartburn

Copyright $\odot$ Iran University of Medical Sciences

Cite this article as: Fakhre Yaseri H. Gender is a risk factor in patients with gastroesophageal reflux disease. Med J Islam Repub Iran. 2017 (8 Sep);31:58. https://doi.org/10.14196/mjiri.31.58

\section{Introduction}

Gastroesophageal reflux disease (GERD) is a recurrent disease that has been defined in the Montreal Consensus Report as a chronic condition that develops when the reflux of gastric contents into the esophagus in significant quantities causes troublesome symptoms with or without mucosal erosions and/or relevant complications (1). The actual prevalence and incidence of reflux is unknown, but it was first reported in Western countries. However, this trend is now extending worldwide. In contrast to previous reports, GERD has become more prevalent in Asian populations in the recent decades (2). For several years, GERD was synonymous with esophagitis and hiatal hernia; it alters quality of life and affects daily activities. The diagnosis of GERD is made using a combination of symptoms

Corresponding author: Dr Hashem Fakhre Yaseri, hfyaseri@yahoo.com

1. Gastrointestinal and Liver Diseases Research Center, \& Department of Internal Medicine, Firoozgar Hospital, Iran University of Medical Sciences, Tehran, Iran. (heartburn and regurgitation) and objective testing associated with endoscopy. The range of GERD prevalence is estimated to be $18.1 \%$ to $27.8 \%$ in North America, $8.8 \%$ to $25.9 \%$ in Europe, $2.5 \%$ to $7.8 \%$ in East Asia, and $8.7 \%$ to $33.1 \%$ in the Middle East (3). Erosive esophagitis has been associated with complications such as esophageal strictures and Barrett's esophagus $(1,4)$; in contrast, the nonerosive reflux disease (NERD) is more associated with changes in patients' quality of life (5). Kim et al. reported that erosive esophagitis was more common in males (11\%) than in females. In addition, they revealed that NERD was more common in females than in males (6). The results of a meta-analysis revealed that the male to female ratio was 1.57 in the prevalence of reflux esopha-

$\uparrow$ What is "already known" in this topic:

For several years, gastroesophageal reflux disease (GERD) was synonymous with esophagitis; GERD alters quality of life and affects daily activities. The frequency of gender and age in patients with GERD in Iranian patients is still unknown.

$\rightarrow$ What this article adds:

This study revealed that erosive reflux disease (ERD), GERD, was more common in Iranian females than in the males. Gender and hiatal hernias were the 2 potential risk factors of GERD. 
gitis (7). The present study aimed at determining the frequency of sex and age in patients with gastroesophageal reflux disease in Iranian patients.

\section{Methods}

\section{Patients}

This study was conducted on 803 patients (11-84 years) with gastroesophageal reflux disease (GERD) during September 2012 and September 2016. GERD was defined as having any degree of reflux esophagitis on endoscopy, or having heartburn or regurgitation on a weekly basis during the last 3 months. The participants were selected from those who referred to the endoscopy ward of Firoozgar hospital. All the patients provided informed consent and accepted to complete a standard questionnaire about heartburn and regurgitation (1, 3, and 5). Esophagogastroduodenoscopy (EGD) was done for all the patients in the same center by expert endoscopists. Reflux esophagitis was diagnosed and graded using Los Angeles classification. Patients with heartburn, regurgitation, and esophagitis were included. The exclusion criteria were as follow: history of malignant diseases, previous foregut surgery, cardiovascular diseases, pregnancy, breastfeeding, psychiatric illness, history of alcohol or drug abuse, large hiatal hernia, and esophageal varices. Moreover, those with achalasia in barium swallow or EGD were excluded. The presence of hiatal hernia was endoscopically diagnosed if the distance from the esophagogastric junction to the diaphragmatic impingement was more than $1 \mathrm{~cm}$. The esophagogastric junction is defined as the proximal margin of the gastric mucosal fold.

\section{Statistical analysis}

Data were analyzed using SPSS18. Age was reported as mean \pm standard deviation. Female patients with gastroesophageal reflux disease were compared with the male patients with respect to the following factors: age, symptoms, esophageal injury (erosive esophagitis), and hiatus hernia. The results were expressed as odds ratios (ORs), with 95\% confidence interval (CIs). Statistical significance was compared between the symptom categories using the Mantel-Haenszel chi-squared test either with Yates correction, or the Fisher's exact probability test. A p value of less than 0.05 was considered statistically significant.

\section{Results}

This study was conducted on 803 patients who met our inclusion criteria. The mean age of the patients was $40.7 \pm 10.7$ years (range: $11-84$ years), and of them $60.5 \%$ (486/803) were female. The mean age of the female and male patients was $39.6 \pm 9.8$ years (age range: $16-84$ years) and 41.5 \pm 8.6 years (age range: 11-79), respectively. The patients younger than 50 years $(69.2 \%(556 / 803)$ were more frequently affected by GERD compared to those older than 50 years [30.8(247/803)]. Of those patients younger than 50 years, $58.8 \%(327 / 556)$ were female. GERD was more prevalent in female patients older than 50 years [64.4(159/247)] than in males [35.6(88/247)], but the difference was not significant $(\mathrm{P}=0.89)$. Of the patients, $84.2 \%(676 / 803)$ and $74.1 \%(595 / 803)$ had heartburn and regurgitation, respectively. Gender (female and male) difference was not a significant factor in heartburn and regurgitation symptoms $(p=0.31$ and $p=0.32$, respectively). Esophageal injuries (esophagitis) and Erosive reflux disease (ERD) were positive in $31.0 \%$ (248/803) of the patients. Gender difference was not significant in ERD patients $(p=0.24)$. Moreover, of the total patients with GERD, 69.0\% (555/803) had normal esophagus, also called nonerosive reflux disease (NERD). NERD was more prevalent in females $(75.3 \%(366 / 486)$ than in males (59.6(189/317, $\mathrm{p}=0.04)$. The female to male ratio in ERD and NERD patients was $1 / 1.06$ and $1.94 / 1$, respectively. Of the patients, $47.9 \%(385 / 803)$ had hiatal hernia in various grades. Hiatal hernia was slightly more prevalent in females $(52.4 \%(202 / 385))$ than in males $(47.6(183 / 385))$, but the difference was not significant $(p=0.36)($ Table 1$)$.

\section{Discussion}

To the best of our knowledge, this was the first Iranian study to date to examine the relationship between sex differences (male and female) and gastroesophageal reflux disease (GERD). The results of the present study revealed that erosive esophagitis (EE) was more common in males than in females (2). However, nonerosive reflux disease (NERD), GERD, was more common in females than in males as it was previously reported (6). The female to male

Table 1. The Demographic Information of the Patients with Gastroesophageal Reflux Disease

\begin{tabular}{|c|c|c|c|c|}
\hline \multirow[t]{2}{*}{ Findings } & \multirow{2}{*}{$\begin{array}{c}\text { Total } \\
\mathrm{N}(\%)\end{array}$} & \multicolumn{2}{|c|}{$\operatorname{Sex}(\mathrm{N}=803)$} & \multirow[b]{2}{*}{$\mathrm{p}$} \\
\hline & & $\begin{array}{c}F \\
N(\%)\end{array}$ & $\begin{array}{c}M \\
N(\%)\end{array}$ & \\
\hline Patients, n (\%) & 803 & $486(60.5)$ & $317(39.5)$ & - \\
\hline \multicolumn{5}{|l|}{ Age(years) } \\
\hline Mean \pm SD & $40.70 \pm 10.7$ & $39.6 \pm 9.8$ & $41.5 \pm 8.6$ & - \\
\hline Range & 11-84 & $16-84$ & 11-79 & \\
\hline \multicolumn{5}{|l|}{ Age(years) } \\
\hline$<50$ & $556(69.2)$ & $327(67.2)$ & $229(72.2)$ & 0.26 \\
\hline$>50$ & $247(30.8)$ & $159(32.8)$ & $88(27.8)$ & 0.89 \\
\hline \multicolumn{5}{|l|}{ Symptomes } \\
\hline Heartburn & $676(84.2)$ & $390(80.2)$ & $286(90.2)$ & 0.31 \\
\hline Regugitation & $595(74.1)$ & $359(73.9)$ & $236(74.4)$ & 0.32 \\
\hline \multicolumn{5}{|l|}{ EGD } \\
\hline EE & $248((31.0)$ & $120(24.7)$ & $128(40.4)$ & 0.24 \\
\hline $\mathrm{NE}$ & $555(69.0)$ & $366(75.3)$ & $189(59.6)$ & 0.04 \\
\hline $\mathrm{HH}$ & $385(47.9)$ & $202(41.6)$ & $183(57.7)$ & 0.36 \\
\hline
\end{tabular}


ratio was 1/1.06 and 1.94/1 in ERD and NERD patients, respectively (7). GERD occurs more in females than in males with an increase in age. The frequency of GERD in patients older than 50 years was associated with increase in age. Heartburn and regurgitation were prevalent in our population as it was previously reported (8), but sex difference was not significant in neither of the symptoms.

Gastroesophageal reflux disease (GERD) is a chronic recurrent disease that develops when the reflux of gastric contents into the esophagus in significant quantities causes troublesome symptoms with or without mucosal erosions and/or relevant complications (1). GERD is a very common disorder with increasing prevalence. The prevalence of GERD varies in different countries. It is estimated that up to $20 \%$ to $25 \%$ of Americans experience symptoms of GERD weekly. Refractory GERD is an increasingly prevalent condition and it has become a major challenge for the clinicians. Traditionally, based on esophagogastric duodenoscopy, patients with GERD could be subdivided into reflux esophagitis (erosive GERD) and endoscopynegative reflux disease (nonerosive reflux disease, NERD) $(1,5)$. Erosive GERD has been associated with complications such as esophageal strictures and Barrett's esophagus (1). The proton pump inhibitors (PPIs) are the drugs of choice in the treatment of GERD (5) because symptoms and pathophysiologic changes to the esophageal mucosa are the results of the effect exposure of the distal esophagus to acidic gastric contents after episodes of gastroesophageal reflux (4).

Several factors are associated with GERD, which are as follow: constitutional factors (eg, age, sex, and obesity); esophageal function (esophageal dysmotility); anatomical abnormalities (gastroesophageal hernias); and environmental factors such as type of diet and eating habits (Westernization of diets); Helicobacter pylori infection treatment and larger elderly population. The most commonly used procedures to diagnose GERD include esophageal multichannel intraluminal impedance-pH (MII-PH) monitoring. However, this technique has some strengths and limitations that the clinicians and investigators should be aware of when deciding to use it (9). The results of this study revealed that the GERD and NERD were more prevalent in females and their frequency increased by increase in age. Such findings may be due to female hormones and hiatal hernia. Kahrilas suggested that GERD is prevalent in pregnant women because progesterone and estrogen hormones can reduce LES pressure and cause GERD in these women (10).

Some researchers reported that hiatal hernias are present in more than $90 \%$ of the patients with severe erosive esophagitis and that they promote GERD chronicity via anatomic changes (10). This study revealed that hiatal hernia was slightly prevalent in females than in males (2), but the difference was not significant. This study had some limitations. First and foremost was that heartburn had overlap symptoms with noncardiac chest pain (NCCP) despite all the cardiovascular workups. Second, the endoscopic findings were reported by 2 or 3 endoscopists in this study, so interobserver conflicts could have occurred.

\section{Conclusion}

In conclusion, the findings of this study revealed that erosive reflux disease (NERD), GERD, was more common in females than in males in the Iranian population. The female to male ratio was $1 / 1.06$ and $1.94 / 1$ in ERD and NERD patients, respectively. GERD occurred more in females than in males with increase in age; therefore, the frequency of GERD in patients older than 50 years was associated with increase in age. Hiatal hernias and gender were the 2 potential risk factors of GERD. Undoubtedly, the findings of the present study should be further analyzed by conducting more studies with larger sample sizes.

\section{Acknowledgments}

We express our appreciation to the Departments of Gastroenterology, Endoscopy, Pathology and the Motility Disorders Laboratory of Firoozgar Teaching Hospital. This study was approved and supported by the Research Center of Iran University of Medical Sciences (Code: 656; Acceptance Ethical No.: IR.IUMS.REC.1394.656).

\section{Conflict of Interests}

The authors declare that they have no competing interests.

\section{References}

1. Vakil N, van Zanten SV,Kahrilas P, Dent J, Jones R;Global Consensus Group. The Montreal definition and classification of gastroesophageal reflux disease: a global evidence-based consensus. Am J Gastroenterol. 2006;101(8):1900-43.

2. Adachi K, Mishiro T, Tanaka S, Hanada K, Kinoshita Y. Gender Differences in the Time-Course Changes of Reflux Esophagitis in Japanese Patients. Intern Med. 2015;54:869-73.

3. Lee SW, Lien HC, Lee TY, Yang SS, Yeh HJ, Chang CS. Heartburn and regurgitation have different impacts on life quality of patients with gastroesophageal reflux disease. World J Gastroenterol. 2014; 20(34):12277-82.

4. Bredenoord AJ, Weusten BLAM, Curvers WL, Timmer R, Smout AJPM. Determinants of perception of heartburn and Regurgitation. Gut. 2006;55:313-18.

5. Moayyedi P, Talley NJ. Gastro-oesophageal reflux disease. Lancet. 2006;367(9528):2086-100.

6. Kim N, Lee SW, Cho SI, Park CG, Yang CH, Kim HS, et al. The prevalence of and risk factors for erosive oesophagitis and nonerosive reflux disease: a nationwide multicentre prospective study in Korea. Aliment Pharmacol Ther. 2008;27:173-85.

7. Ford AC, Forman D, Reynolds PD, Cooper BT, Moayyedi P. Ethnicity, Gender and Socioeconomic Status as Risk Factors for Esophagitis and Barrett's Esophagus. Am J Epidemiol. 2005;162(5):454-60.

8. Yaseri HF, Yaseri AMF, Moghaddam AB, Arabshhi SKS. Relationship between Esophageal Clinical Symptoms and Manometry Findings in Patients with Esophageal Motility Disorders: A CrossSectional Study. Med J Islam Repub Iran. 2015;29:271.

9. Savarino E, Pohl D, Zentilin P, Dulbecoo P, Sammito G, Sconfienza $\mathrm{L}$, et al. Functional heartburn has more in common with functional dyspepsia than with non-erosive reflux disease. Gut. 2009;58:118591.

10. Rey E, Artalejo FR, Herraiz MA, Puy Sanchez, Sanchez AA, Escudero M, et al. Gastroesophageal Reflux Symptoms During and After Pregnancy: A Longitudinal Study. Am J Gastroenterol. 2007; 102:2395-400. 